2020, Band 8, Heft 3

Seiten 151-156

zeitschrift-suburban.de

10.36900/suburban.v8i3.643

\title{
Den Osten im Blick
}

Matthias Bernt, Andrej Holm: ,Die Ostdeutschlandforschung muss das Wohnen Eine Replik in den Blick nehmen'

Kommentare von: Barbara Schönig, Carsten Keller, Carmen Leidereiter, Dieter Rink, Felix Ringel

Replik von:

Mathias Bernt

Matthias Bernt, Andrej Holm

Andrej Holm

Die Kommentare zu unserem „Plädoyer für eine neue politisch-institutionelle Perspektive auf ostdeutsche Städte" lassen sich grob in zwei Argumentationslinien einteilen: Das ist zum einen eine Perspektive, die Zweifel an unserem politökonomisch-institutionellen Ansatz sowie der Eignung von Ostdeutschland als eigenständigem Forschungsgegenstand anmeldet, sowie zweitens eine Gruppe an Argumenten, die unseren Ansatz unterstützt - aber nach den forschungspolitischen Konsequenzen fragt. Beide Linien lassen sich dabei nicht eindeutig bestimmten Autor_innen zurechnen. In unserer Replik können wir natürlich nicht auf alle Anregungen eingehen. Wir beschränken uns daher auf die Themen, die in mehreren Kommentaren aufgegriffen wurden.

Barbara Schönig hinterfragt, „[...] was und wer [...] mit welchem Recht als ,ostdeutsch“ zu betrachten wäre“ und „ob und inwieweit Forschung die nach Differenzen in einem vielfach verwobenen Feld gesellschaftlicher und räumlicher Entwicklung in Deutschland sucht, gerade diese nicht auch stetig neu verfestigt und damit die Pluralität ostdeutscher Entwicklung und Erfahrungen und gegebenenfalls auch Parallelitäten zu Westdeutschland überschreibt." (Schönig 2020: 116) Sie führt dabei drei Gründe an, die sie gegenüber einer, Ostdeutschlandforschung' skeptisch machen: Erstens sieht sie Schwierigkeiten dabei, nach 30 Jahren gemeinsamer Geschichte überhaupt noch zu bestimmen, wer als ,ostdeutsch' gelten kann. Angesichts der vielfachen Pendelbiographien ist das in einem gewissen Maß nachvollziehbar. Zweitens deutet Barbara Schönig, wie auch Dieter Rink in seinem Text, auf die Binnendifferenzierung in Ostdeutschland hin. Auch hier können wir zustimmen: Natürlich ist die Situation in Jena nicht dieselbe wie in Suhl (genauso wie sich auch München und Gelsenkirchen unterscheiden). Drittens verweist sie auf die Gemeinsamkeiten zwischen abgehängten und benachteiligten Regionen. Auch hier können wir zustimmen: ,Verlierer' von Globalisierung und Neoliberalismus gib es selbstverständlich nicht nur in der Niederlausitz oder in Halle-Neustadt, sondern auch im Ruhrgebiet, im US-amerikanischen, rustbelt' oder in Nordengland.

Insgesamt finden wir diese Einwände also gut nachvollziehbar. Nichtsdestotrotz glauben wir, dass sie am Kern des Problems vorbeigehen. Um das 
zu verdeutlichen, möchten wir uns einen kurzen Ausflug in die Diskussion über „ordinary cities“ (Robinson 2006) und die damit eng verbundenen Diskussionen über adäquate Wege zur Untersuchung postsozialistischer Städte (Grubauer/Kusiak 2012; Ferenčuhová 2012; Tuvikene 2016; Golubchikov et al. 2014) erlauben, von der die deutsch-deutsche Debatte unserer Meinung nach viel lernen kann. In dieser Debatte ist ein Verständnis von postsozialistischen Städten als „spatio-temporal container(s)“ (Tuvikene 2016) inzwischen weitgehend überwunden. Ein solches Verständnis dominierte lange Zeit die Forschung und es basierte auf einer klaren Vorstellung zu den Parametern, die postsozialistische Städte ausmachen: (1) sie befinden sich im ehemaligen Ostblock, (2) die zeitliche Dimension der Analyse bezieht sich auf die Jahre seit der ,Wende', (3) der zu beobachtende Wandel lässt sich als eine Transition verstehen, der diesen Ort vom Sozialismus zum Kapitalismus führt. Inzwischen ist es in der internationalen Debatte Konsens, dass eine solche Perspektive defizitär ist.

Im Gegensatz zu dieser Konzeptionalisierung betonen heute viele Beiträge ein Verständnis von Postsozialismus als ,condition'. Dabei werden drei Aspekte als zentral aufgefasst: (1) werden postsozialistische Gesellschaften als Hybride aufgefasst, die Kontinuitäten und Brüche vereinen; (2) werden Verbindungen zwischen Orten und Räumen betont - die Entwicklung von Städten im ehemaligen Ostblock wird also als eingebettet in Netzwerke von Akteuren und Flüsse von Ideen, Menschen und Kapital gedacht, die diese mit anderen Räumen verbinden; (3) schließlich hat sich diese Debatte weitgehend von der Vorstellung einer Transition verabschiedet, die nur in eine Richtung geht und anstatt dessen die vielfachen Kombinationen unterschiedlicher Transformationen betont, die unterschiedliche soziale Logiken miteinander verbinden und zu multiplen räumlich spezifischen Arrangements führen.

Im Licht dieser Debatten kann es also gar nicht darum gehen, ostdeutsche Städte in einer wesenshaften Einzigartigkeit zu entschlüsseln und ihnen eine räumlich-zeitlich genau definierte Nische zuzuschreiben. Im Gegenteil muss die Forschung über ostdeutsche Städte Kontinuitäten, Brüche und Verbindungen zusammen denken. Eine Stadt wie Cottbus ist so gleichzeitig post-sozialistisch, deindustrialisiert, neoliberal, schrumpfend und zunehmend kulturell divers. Während einige dieser Aspekte eher zu einem Vergleich von Cottbus mit Ostrava anregen würden, legen andere Aspekte möglicherweise eher einen Vergleich mit Sheffield nahe. Selbst eine Analyse der Spannungsverhältnisse, die sich aus der zunehmenden Diversität der Cottbusser Einwohnerschaft ergeben, könnte aber kaum ohne ein tieferes Verständnis der DDR- und Nachwendeerfahrungen auskommen. Anders gesagt, eine Ostdeutschlandforschung muss natürlich zu einem relationalen und de-territorialisierten Verständnis von Ostdeutschland finden - aber indem sie dies tut, benötigt sie auch ein Wissen um die Spezifik ihres Forschungsgegenstandes. Entsprechend hat auch die von Dieter Rink hervorgehobene Schrumpfungsforschung ganz sicher zu einem tieferen Verständnis der spezifischen Bedingungen für die Stadtentwicklung in Ostdeutschland beigetragen. Sie blieb jedoch eher Schrumpfungs- als Ostdeutschlandforschung und konnte so nur wenig Impulse für die über das Thema Schrumpfung hinausgehende Auseinandersetzung mit Ostdeutschland setzen. Auch hier 
veränderte die Fülle an Fallstudien kaum etwas am Status Ostdeutschlands als Nischenthema.

Eng verwandt mit dieser Problematik sind aus unserer Sicht Carsten Kellers Anmerkungen zum Verhältnis von Empirie und Konzeptionalisierung. Möglicherweise haben wir uns hier falsch ausgedrückt. Uns geht es natürlich nicht darum, dass kein empirisches Material zu Ostdeutschland mehr erhoben werden sollte. Die Erfahrung der letzten Jahrzehnte hat aber eben auch gezeigt, dass die Prognosekraft vieler Untersuchungen gering war.

So erwiesen sich die Vorhersagen zu einer Angleichung des Parteiensystems und der Wahlpräferenzen in Ostdeutschland an das westdeutsche Referenzsystem (z. B. Niedermeyer/Stöss 1994: 27) ebenso als Fehleinschätzung wie Prognosen zu einer Anpassung der Wirtschaftsentwicklung in Ostdeutschland an das Westniveau (Brenke et al. 1999: 14) oder die von Dieter Rink zitierte These einer Wiedereinordnung der ostdeutschen Städte in die historische Städtehierarchie (Henckel et al. 1993). Auch viele Einschätzungen von Stadtentwicklungstrends hatten in der Abfolge „nachholende Suburbanisierung“, „Schrumpfung“, „Reurbanisierung“ und „verstärkte Umlandorientierung“ in den letzten 30 Jahren nur jeweils kurze Halbwertzeiten (Siedhoff 2020: $266 \mathrm{ff}$.)

Aus unserer Sicht verweist das auf Schwächen der Konzepte, die offensichtlich nicht in der Lage waren, Orientierung zu bieten, mittels derer Untersuchungen besser angeleitet werden könnten. Wir möchten dies am Beispiel der Gentrification-Forschung erläutern: Hier sind seit 1990 wirklich viele Untersuchungen in ostdeutschen Städten angestellt worden (vgl. Üblacker 2018), wahrscheinlich sogar mehr als im Westen. Die überwiegende Zahl der Studien folgte dabei einem von Blasius, Friedrichs und Dangschat auf der Grundlage von Hamburg und Köln entwickelten Modell eines doppelten Invasions-Sukzessions-Zyklus. Dabei wurde immer wieder festgestellt, dass dieses Modell nur mit Abstrichen auf ostdeutsche Städte angewendet werden kann (vgl. Bernt/Rink/Holm 2010). Angesichts dieses Befunds hätte man sich entweder fragen können, ob die vorhandenen Erklärungen zu Gentrification modifiziert werden müssen oder ob die Andersartigkeit ostdeutscher Gentrification-Prozesse etwas über die Andersartigkeit ostdeutscher Städte allgemein aussagt. In jedem Fall hätte die Feststellung empirischer Besonderheiten zur konzeptionellen Neuorientierung geführt. Dass eine solche tatsächlich unterblieben ist, halten wir für problematisch. Es geht uns also nicht um eine Gegenüberstellung von Theorie und Empirie, sondern um die Frage, ob ein Ineinandergreifen von empirischer Forschung und theoretischer Konzeptionalisierung zustande kommt, bei dem Konzepte empirische Forschung informieren, empirische Forschung zur Überprüfung und Reformulierung von Konzepten eingesetzt wird und neue Konzepte wiederum mittels neuer empirischer Forschung überprüft und hinterfragt werden können. Wir möchten unser Plädoyer für konzeptionelle Überlegungen also keineswegs als Absage an die empirische Forschung verstanden wissen.

Vor dem Hintergrund der anhaltenden Schwierigkeiten, den Gegenstand einer Ostdeutschlandforschung zu bestimmen, fanden wir die Überlegungen von Felix Ringel sehr anregend, der die Politiken der Ostdeutschlandforschung in den Blick nimmt. Im Kern stellt Felix Ringel dabei die Frage, ob die Marginalisierung der Ostdeutschlandforschung Ost-West-Machtverhältnisse im 
Wissenschaftsbetrieb reproduziert und entwickelt auf dieser Basis den Vorschlag eines „strategischen Essentialismus“ (Ringel 2020: 149). Wir finden, dass das ein sehr interessanter Vorschlag ist, der gleichwohl Ecken und Kanten hat.

Zunächst erscheint uns dabei selbstverständlich, dass die Forschung zu Ostdeutschland - ähnlich wie das Feld der ,Area Studies“ von einer regionalen Expertise getragen werden sollte, die es ermöglicht, Entwicklungen in diesem Teil Deutschlands auf einer tieferen Basis zu verstehen. Wenn immer wieder neu erarbeitet werden muss, was zum Beispiel die Restitution oder das Altschuldenhilfegesetz war und welche Folgen beide hatten (um bei unseren Beispielen zu bleiben) und wenn Debatten der vergangenen Jahrzehnte nicht bekannt sind, wird es in der Tat schwierig, irgendeinen Fortschritt zu erreichen.

Viel wichtiger ist aus unserer Ansicht aber das in Ringels Überlegungen implizit formulierte Ziel einer Infragestellung vorhandener "geographies of theory“ (Roy 2009). Hier stimmen wir zu, dass ein besseres, feinkörniges und nuanciertes Wissen über Ostdeutschland nicht nur die Qualität der Forschung insgesamt verbessern würde, sondern es vor allem auch leichter machen könnte, Fragen zu stellen und Überlegungen anzubieten, die von gesamtdeutschem Interesse sind. Was sagt es beispielsweise über die Reproduktion von Klassenstrukturen aus, wenn Westdeutsche in typischen Gentrifizierungsgebieten in Ostdeutschland überrepräsentiert, hingegen in Großwohnsiedlungen unterrepräsentiert sind? Warum spielen Ostdeutsche in Entscheidungseliten auch in Ostdeutschland kaum eine Rolle? Welche Rückschlüsse ermöglicht die geringere Akzeptanz der bundesrepublikanischen, freiheitlich-demokratischen Grundordnung' in Ostdeutschland nicht nur auf die Subjektivitäten der Ostdeutschen, sondern über die Substanz dieser Grundordnung? In all diesen Fragen hätte eine selbstbewusste Ostdeutschlandforschung das Potenzial, nicht allein Ostdeutschland sichtbarer zu machen, sondern neue Fragen aufzuwerfen, die auch über den Gegenstand ihrer Forschung hinaus wissenschaftlich und politisch wichtig sind.

Um diese Fragen aufwerfen zu können, muss sich die Ostdeutschlandforschung allerdings zunächst einen Raum schaffen. Der Verweis auf einen „strategischen Essentialismus“(Spivak 1988) als politische und intellektuelle Taktik erscheint uns hier produktiv. Die wiederkehrende Infragestellung der Berechtigung einer Forschung zu Ostdeutschland, ihre symbolische, finanzielle und institutionelle Marginalisierung und die Stärke vorhandener Zuschreibungen erzwingen geradezu eine Praxis, in der sich unterschiedliche Ansätze um ,Ostdeutschland' als Forschungsgegenstand herum versammeln, um gemeinsam ihre Widerstands- und Handlungsfähigkeit gegenüber einem hegemonialen westdeutschen Diskurs zu verbessern. Dabei wird alleine eine „Vernetzung“ der „kleinen Ostdeutschlandforschung“( Rink 2020: 142) nicht ausreichen. Der faktischen Unterrepräsentation des Forschungsgegenstandes muss auch eine selbstbewusstere Etikettierung der Ostdeutschlandforschung entgegengesetzt werden.

Gleichzeitig sehen wir hier zwei Schwierigkeiten: Erstens muss ein Verständnis von ,Ostdeutschland“ - wie wir oben gezeigt haben - notwendig relational, de-territorialisiert und anti-essentialistisch operieren. Eine Ostdeutschlandforschung steht damit vor der Herausforderung, ihren 
Gegenstand gleichzeitig zu fixieren und zu dekonstruieren. Zweitens würde ein solcher ,strategischer Essentialismus unter den gegebenen Bedingungen aus einer Position der relativen Schwäche operieren. Ohne Zugang zu Forschungsmitteln, mit geringem innerwissenschaftlichem Renommee und schwachen Karrieremöglichkeiten ausgestattet, erscheint uns die materielle Basis dieser Orientierung als außerordentlich dünn. Der Vorschlag von Felix Ringel läuft daher auf eine paradoxe Problematik hinaus: die unmögliche und gleichzeitig notwendige Selbstrepräsentation von ,Ostdeutschland' in einer gesamtdeutschen (und globalen) Landschaft der Wissensproduktion.

Die Entwicklungsmöglichkeiten, die eine solche Fokussierung nichtsdestotrotz bietet, werden in paradigmatischer Weise im Kommentar von Carmen Leidereiter aufgezeigt. Wir kannten diese Forschung bislang nicht, waren aber umso mehr beeindruckt von den Parallelen zu unseren eigenen Überlegungen. Aus unserer Sicht zeigt diese Forschung beispielhaft, wie eine Tiefenbohrung in ostdeutsche Lebensverhältnisse gleichzeitig strukturell und subjektorientiert argumentieren kann. Carmen Leidereiter gelingt es dabei, auch ohne a priori-Klärung des Objektes ihrer Forschung (und ohne Selbstrechtfertigungsrituale) zu zeigen, wie die Wiederkehr längst überwunden geglaubter Ausbeutungsverhältnisse, die geringen Handlungsmöglichkeiten Ostdeutscher und die „affektiven Spuren“ (Leidereiter 2020: 129) auf der Ebene der Subjektivitäten in Vorpommern zusammenhängen. Von solchen Beiträgen wünschen wir uns mehr.

Die Publikation dieses Beitrags wurde durch Mittel des Open-Access-Publikationsfonds des Leibniz-Instituts für Raumbezogene Sozialforschung gefördert.

\section{Autor_innen}

Matthias Bernt ist Politologe. Er arbeitet zu Fragestellungen urbaner Governance, zu Gentrification, schrumpfenden Städte, sowie zu Migration und Regenerierung.

matthias.bernt@leibniz-irs.de

Andrej Holm ist Sozialwissenschaftler und arbeitet u.a. zu Gentrifizierung, Wohnungspolitik und städtischen Protestbewegungen.

a.holm@sowi.hu-berlin.de

\section{Literatur}

Bernt, Matthias / Rink, Dieter / Holm, Andrej (2010): Gentrificationforschung in Ostdeutschland: Konzeptionelle Probleme und Forschungslücken. In: Berichte zur deutschen Landeskunde 84/2, 185-203.

Brenke, Karl/ Eickelpasch, Alexander/ Postlep, Rolf-Dieter/ Gerling, Katja/ Sander, Birgit/ Schmidt, Klaus-Dieter/ Beer, Siegfried/ Bergemann, Annette/ Komar, Walter/ Müller, Ralf/ Ragnitz, Joachim/ Traben, Lioba (1999): Gesamtwirtschaftliche und unternehmerische Anpassungsfortschritte in Ostdeutschland (Neunzehnter Bericht). Kieler Diskussionsbeiträge No. 346/347. Kiel: Institut für Weltwirtschaft (IfW).

Ferenčuhová, Slavomira (2012): Urban theory beyond the „East/West divide“? Cities and urban research in postsocialist Europe. In: Tim Edensor / Mark Jayne (Hg.), Urban theory beyond the West: a world of cities. Routledge: London/New York, 65-74. 
Golubchikov, Oleg/ Badyina, Anna / Makhrova, Alla (2014): The Hybrid Spatialities of Transition: Capitalism, Legacy and Uneven Urban Economic Restructuring. In: Urban Studies 5/4, 617-633.

Grubbauer, Monika / Kusiak, Joanna (2012): Introduction: Chasing Warsaw. In: Monika Grubbauer / Joanna Kusiak (Hg.), Chasing Warsaw: socio-material dynamics of urban change since 1990. Frankfurt/New York: Campus, 9-24.

Henckel, Dietrich/ Grabow, Busso / Hollbach, Beate/ Usbeck, Hartmut/ Niemann, Heinz (1993): Entwicklungschancen deutscher Städte. Die Folgen der Vereinigung. Stuttgart u. a.: Kohlhammer.

Leidereiter, Carmen (2020): Rückschritte in längst überwunden geglaubte Ausbeutungs- und Unsicherheitsmuster. Kommentar zu Matthias Bernt und Andrej Holm „Die Ostdeutschlandforschung muss das Wohnen in den Blick nehmen“. In: sub \urban. zeitschrift für kritische stadtforschung 8/3, 129-136.

Niedermayer, Oskar / Stöss, Richard (1994): DDR-Regimewandel, Bürgerorientierungen und die Entwicklung des gesamtdeutschen Parteiensystems. In: Oskar Niedermayer / Richard Stöss, (Hg.), Parteien und Wähler im Umbruch. Opladen: Westdeutscher Verlag, 11-33.

Ringel, Felix (2020): Zur Zukunft der Ostdeutschlandforschung. Kommentar zu Matthias Bernt und Andrej Holm „Die Ostdeutschlandforschung muss das Wohnen in den Blick nehmen“. In: sub \urban. zeitschrift für kritische stadtforschung 8/3, 145-150.

Rink, Dieter (2020): Schrumpfung und Stadtumbau als zentrale Themen der Forschung zu Städten in Ostdeutschland. Kommentar zu Matthias Bernt und Andrej Holm „Die Ostdeutschlandforschung muss das Wohnen in den Blick nehmen“. In: sub \urban. zeitschrift für kritische stadtforschung 8/3, 137-144.

Robinson, Jennifer (2006) Ordinary cities: between modernity and development. London/ New York: Routledge.

Roy, Ananya (2009): The 21st-Century Metropolis: New Geographies of Theory. In: Regional Studies 43/6, 819-830.

Schönig, Barbara (2020): Ererbte Transformation. Kommentar zu Matthias Bernt und Andrej Holm „Die Ostdeutschlandforschung muss das Wohnen in den Blick nehmen“. In: sub \urban. zeitschrift für kritische stadtforschung 8/3, 115-122.

Siedhoff, Mattias (2020): Vorzeichenwechsel der Stadtentwicklung in Ostdeutschland. In: Sören Becker / Matthias Naumann (Hg.), Regionalentwicklung in Ostdeutschland. Dynamiken, Perspektiven und der Beitrag der Humangeographie. Wiesbaden: Springer Spektrum, 281-294.

Spivak, Gayatri Chakravorty (1988): Can the Subaltern Speak? In: Cary Nelson / Lawrence Grossberg (Hg.), Marxism and the Interpretation of Culture. Chicago: University of Illinois Press, 271-313.

Tuvikene, Tauri (2016): Strategies for Comparative Urbanism: Post-socialism as a De-territorialized Concept. In: International Journal of Urban and Regional Research 40, 132-146.

Üblacker, Jan (2018): Gentrifizierungsforschung in Deutschland. Eine systematische Forschungssynthese der empirischen Befunde zur Aufwertung von Wohngebieten. Opladen u. a.: Budrich. 